OS-GE-34-93

RCNP-057

July 1993

\title{
Quantum Deformation of the Affine Transformation Algebra
}

\author{
N. Aizawa* \\ Research Center for Nuclear Physics \\ Osaka University, Ibaraki, Osaka 567, Japan \\ and \\ H.-T. Sato ${ }^{\dagger}$ \\ Institute of Physics, College of General Education \\ Osaka University, Toyonaka, Osaka 560, Japan
}

\begin{abstract}
We discuss quantum deformation of the affine transformation algebra. It is shown that the quantum algebra has a non-cocommutative Hopf algebra structure, simple realizations and quantum tensor operators.
\end{abstract}

$\dagger$ Fellow of the Japan Society for the Promotion of Science

E-mail : hsato@jpnyitp.yukawa.kyoto-u.ac.jp

* E-mail : aizawa@rcnpth.rcnp.osaka-u.ac.jp 
The notion of quantum deformation of Lie algebras and the groups has been developed in recent years [1-6]. The quantum groups is characterized by the fact that the elements of its representation matrix do not mutually commute. And the quantum algebras is understood that the universal enveloping algebra has the structure of non-cocommutative Hopf algebra. The deformation of the classical groups and the algebras have been discussed by many authors. The simplest example of the quantum algebra is $U_{q}(s l(2))$ and that of the quantum group $S L_{q}(2)$. Their representation theories have been well investigated. It is also recognized that $U_{q}(s l(2))$ and $S L_{q}(2)$ are dual each other [7, 8]. The deformation of exceptional and super groups and their algebras are also developed [9, 10].

In this paper, we present the quantum deformation of the affine transformation algebra which is one of the fundamental transformations. We suggest that the quantum algebra is possible to be provided by deforming maps from the undeformed algebra. Some examples of the realizations are also shown. The quantum analogue of the tensor operator, which carries the adjoint representation, is explicitly given in terms of the generators of the quantum affine transformation algebra.

The affine transformation means the following operations

$$
\hat{p}=-i \frac{d}{d x}, \quad \hat{d}=\frac{i}{2}\left(x \frac{d}{d x}+\frac{d}{d x} x\right),
$$

where $\hat{p}$ is the momentum operator in one-dimensional quantum mechanics and $\hat{d}$ is the dilatation operator. It is easy to observe that they satisfy the relations

$$
[\hat{p}, \hat{d}]=i \hat{p}
$$

and

$$
\exp (-i a \hat{p}) x \exp (i a \hat{p})=x-a
$$

and

$$
\exp (i \ln b \hat{d}) x \exp (-i \ln b \hat{d})=b x .
$$

In an abstract sense, the affine transformation is the group whose multiplication law is given by

$$
U(a, b) U(\alpha, \beta)=U(a+\alpha b, \beta b) .
$$

The elements of the group can be parametrized by

$$
U(a, b)=\exp (i a p) \exp (-i \ln b d)
$$


where the Lie algebra $A=\{p, d\}$ satisfies the commutation relation

$$
[d, p]=i p
$$

The allowed region of the parameters is $-\infty<a<\infty, 0<b<\infty$, which requires that the group manifold is a half-plane, and so the affine transformation group is a non-Abelian and non-compact group.

The unitary representations of the affine transformation group are found in refs. 12] and [13]. These papers have shown that there are two and only two unitary irreducible representations. One is the case of the positive eigenvalues of $p$ and the other the negative case. For example, if we take the following realization of the algebra $A$ on the space of square integrable functions

$$
p=k, \quad d=(i / 2)\left(k \partial_{k}+\partial_{k} k\right),
$$

the cases of $k>0$ and $k<0$ give inequivalent irreducible representations.

The eigenvalue of $p$ has continuous value in the unitary irreducible representation. In order to have a simple matrix representation, we consider the adjoint representation. The adjoint representation is not a unitary one because it is a finite dimensional representation and the affine transformation group is non-compact. The adjoint representation of the algebra $A$

$$
p=\left(\begin{array}{cc}
0 & 0 \\
-i & 0
\end{array}\right), \quad d=\left(\begin{array}{cc}
0 & 0 \\
0 & i
\end{array}\right),
$$

gives the adjoint representation of the affine transformation group by substitution of (9) into (6)

$$
U(a, b)=\left(\begin{array}{ll}
1 & 0 \\
a & b
\end{array}\right) .
$$

Now, let us present a one-parameter deformation of the universal enveloping algebra of $A$ and the realizations. The quantum affine transformation algebra $U_{q}(A)=\{D, P\}$ is defined by the commutation relation

$$
[D, P]=i[P]
$$

where $[P] \equiv\left(q^{P}-q^{-P}\right) /\left(q-q^{-1}\right)$ and $q$ is as usual a deformation parameter. This is a non-cocommutative Hopf algebra as can be seen as follows. The 
Hopf algebra mappings, coproduct $\Delta$, counit $\epsilon$ antipode $S$, are given by

$$
\begin{gathered}
\Delta(P)=P \otimes 1+1 \otimes P, \\
\Delta(D)=D \otimes q^{-P}+q^{P} \otimes D, \\
\epsilon(P)=\epsilon(D)=0, \\
S(P)=-P, \quad S(D)=-D-i(\ln q)[P],
\end{gathered}
$$

and eqs.(12) certainly satisfy the following axioms of the Hopf algebra

$$
\begin{gathered}
(i d \otimes \Delta) \circ \Delta=(\Delta \otimes i d) \circ \Delta, \\
(i d \otimes \epsilon) \circ \Delta=(\epsilon \otimes i d) \circ \Delta=i d, \\
m(i d \otimes S) \circ \Delta=m(S \otimes i d) \circ \Delta=1 \epsilon,
\end{gathered}
$$

where $i d$ denotes the identity mapping and $m$ the product of the two terms in the tensor product ; $m(x \otimes y)=x y$. If we define the opposite coproduct $\Delta^{\prime}$ by

$$
\Delta^{\prime}=\sigma \circ \Delta, \quad \sigma(x \otimes y)=y \otimes x,
$$

$\Delta^{\prime}(P)$ and $\Delta^{\prime}(D)$ also satisfy the same commutation relation as (11). For the opposite coproduct, $S(P)$ and the counit are not changed while $S(D)$ becomes

$$
S(D)=-D+i(\ln q)[P] .
$$

Next, we show some realizations of the $U_{q}(A)$. The generators $P$ and $D$ can be formally expressed in terms of the undeformed ones

$$
P=p, \quad D=\frac{1}{2}\left(\frac{[p]}{p} d+d \frac{[p]}{p}\right) .
$$

When the representation of $p$ and $d$ in the Hilbert space is considered, $p$ and $d$ are hermite operators. The realization (16) of $P$ and $D$ is also chosen to be hermitian in the same representation space when $q$ is real or $|q|=1$. If we require only satisfying the commutation relation (11), $D$ can be simply given by

$$
D=\frac{[p]}{p} d .
$$

When the representation and the realization of $A$ have the inverse $p^{-1}$ or $[p]$ are proportional to $p$, they can be transformed into those of the $U_{q}(A)$ by making use of (16) or (17). 
Here we show the examples of both cases:

(i) The case of having $p^{-1}$. The realization of eq.(8) is transformed into

$$
P=k, \quad D=\frac{i}{2}\left([k] \partial_{k}+\partial_{k}[k]\right),
$$

it is easy to verify that the commutation relation (11) holds.

(ii) On the other hand, the adjoint representation of the $U_{q}(A)$ cannot be obtained by naive use of the relation (16) since the adjoint representation of the undeformed generators (9) does not have $p^{-1}$. However $[p]$ reduces to be proportional to $p$, i.e.

$$
[p]=\delta p, \quad \delta \equiv \frac{2 \ln q}{q-q^{-1}}
$$

so we get the adjoint representation of $P$ and $D$ with the aid of eq.(16)

$$
P=\left(\begin{array}{cc}
0 & 0 \\
-i & 0
\end{array}\right), \quad D=\left(\begin{array}{cc}
0 & 0 \\
0 & i \delta
\end{array}\right)
$$

The adjoint representation of the $U_{q}(A)$ is the matrices (9) multiplied by $q$-dependent factor $\delta$. The factor $\delta$ becomes unity as $q \rightarrow 1$.

Finally, we give the tensor operator which carries the adjoint representation of the $U_{q}(A)$. The definition of tensor operator of quantum algebras was given by Rittenberg and Scheunert [11] in terms of the representation theory of Hopf algebras. The tensor operator is generally defined through the following adjoint action of the Hopf algebra $H$. The adjoint action of $c \in H$ on $t \in H$ is defined by

$$
a d(c) t=\sum_{i} c_{i} t S\left(c_{i}^{\prime}\right)
$$

where we denote the coproduct of $c$ by

$$
\Delta(c)=\sum_{i} c_{i} \otimes c_{i}^{\prime}
$$

Writing the $n \times n$ matrix representation of $c$ as $\rho_{i j}(c)$, the tensor operators $\left\{T_{i}, i=1,2, \cdots, n\right\}$ which carry the representation $\rho(c)$ are defined by the relation

$$
\operatorname{ad}(c) T_{i}=\sum_{j} \rho_{j i}(c) T_{j}
$$


Namely, the tensor operators $\left\{T_{i}\right\}$ form a representation basis under the adjoint action. Now in the case of the $U_{q}(A)$, we can write down the adjoint action of $P$ and $D$ on $T \in U_{q}(A)$

$$
\begin{gathered}
a d(P) T=[P, T] \\
a d(D) T=D T q^{P}-q^{P} T D-i(\ln q) q^{P} T[P] .
\end{gathered}
$$

We therefore find the tensor operators which carry the adjoint representation of (19) :

$$
\begin{aligned}
& T_{1}=q^{-P} D, \\
& T_{2}=q^{-P}[P] .
\end{aligned}
$$

It is noted that RHS of eq.(22) reduces to the commutators and eq.(23) to $d$ and $p$ in the limit of $q \rightarrow 1$.

In this paper, we showed that the quantum deformation of the affine transformatio algebra is possible. This fact is non-trivial. The reason is that the affine transformation has simple but different structure from the classical Lie algebras. The quantum affine transformation algebra has the non-cocommutative Hopf algebra structure and its deforming map and tensor operators were given explicitly. Since the quantum affine transformation algebra still possesses simple structure, it would be possible to use the quantum affine transformation algebra as building block of other quantum groups and algebras. We can make another algebraic relation which belongs to the present $U_{q}(A)$, i.e. ,

$$
\tilde{D} \tilde{P}-q \tilde{P} \tilde{D}=i \tilde{P}
$$

introducing the recombination

$$
\begin{aligned}
& \tilde{P}=f(\Lambda) P, \\
& \tilde{D}=i q^{(\Lambda-1) / 2}[\Lambda]_{1 / 2},
\end{aligned}
$$

where $f(\Lambda)$ is an arbitrary function of $\Lambda$ provided that $f(\Lambda) \rightarrow 1$ as $q \rightarrow 1$ and

$$
\begin{gathered}
\Lambda \equiv-i \frac{P}{[P]} D, \\
{[\Lambda]_{1 / 2} \equiv \frac{q^{\Lambda / 2}-q^{-\Lambda / 2}}{q^{1 / 2}-q^{-1 / 2}} .}
\end{gathered}
$$


The above q-commutation relation (24)is a linear algebra, which is different from the original nonlinear algebra (11). It is thus possible to investigate linear representations on the linear basis. This subject should be discussed in a separate paper.

\section{References}

[1] V. G. Drinfeld, Quantum Groups, ICM proceedings vol. 1, p798, New York: Berkeley (1986), (The American Mathematical Society 1987).

[2] M. Jimbo, Lett. Math. Phys. 10 (1985) 63 ; 11 (1986) 247 ; Comm. Math. Phys. 102 (1986) 537.

[3] E. K. Sklyanin, Funct. Anal. Appl. 16 (1982) 263.

P.P. Kulish and N. Yu. Reshetikhin, J. Soviet. Math. 23 (1983) 2435.

[4] L. D. Faddeev, N. Yu. Reshetikhin and L. A. Takhtajan, Algebra Anal. 1 (1987) 178.

[5] S. L. Woronowicz, Publ. RIMS. Kyoto univ. 23 (1987) 117 ; Comm. Math. Phys. 111 (1987) 613 ; 122 (1989) 125.

[6] Yu. I. Manin, Quantum gruops and non-commutative geometry, Les publications du Centre de Recherches Mathématiques, Univ. Montréal (1988) ; Comm. Math. Phys. 123 (1989) 163.

[7] L. A. Takhtajan, Lecture Notes Phys. 370 (1990) 3.

[8] S. Majid, Int. J. Mod. Phys. A5 (1990) 1 ; J. Math. Phys. 32 (1991) 3246.

[9] I. G. Koh and Z.-Q. Ma, Phys. Lett. B234 (1990) 480.

L. Frappat, P. Sorba and A. Sciarrino, J. Phys. A24 (1991) L179.

[10] M. Chaichian and P. P. Kulish, Phys. Lett. 234 (1990) 72.

[11] L. C. Biedenharn and M. Tarlini, Lett. Math. Phys. 20 (1990) 271.

M. Nomura, J. Phys. Soc. Jpn. 59 (1990) 439, 2345.

V. Rittenberg and M. Scheunert, J. Math. Phys. 33 (1992) 436. 
[12] I. M. Gel'fand and M. A. Naimark, Dokl. Akad. Nauk SSSR, 55 (1947) 570.

[13] E. W. Aslaksen and J. R. Klauder, J. Math. Phys. 9 (1968) $206 ; 10$ (1969) 2267. 